\title{
Retreat of the West Antarctic Ice Sheet from the western Amundsen Sea shelf at a pre- or early LGM stage:
}

\section{Constraining Antarctic LGM glaciation from nearshore records}

\begin{abstract}
Defining the extent of the Last Glacial Maximum Antarctic lce Sheet and the timing of its subsequent retreat still remains poorly understood for numerous drainage sectors. New marine geoscientific field data from a formerly unstudied West Antarctic continental shelf sector reveal the last maximum extent of the ice sheet and its initial retreat. It is shown how modern continental shelf regions in the study area must have already been evacuated at a remarkably early stage, thereby validating a rather diachronous retreat pattern of the Antarctic lce Sheet following its last maximum extent.
\end{abstract}

\section{Key findings}

- Inner shelf deglaciated by 20.88 cal. ka BP - Shelf-wide grounded ice cover during Local Last Glacial Maximum (LLGM) prior to 20.88 cal. ka BP - shallow glacial unconformity Ice sheet stabilization on inner shelf - deposition of large grounding-zone wedge (GZW) caused by change in subglacial substrate Retreat rate of $<2 \mathrm{~m} / \mathrm{a}$ in the past $\sim 21 \mathrm{ka}$ (b)
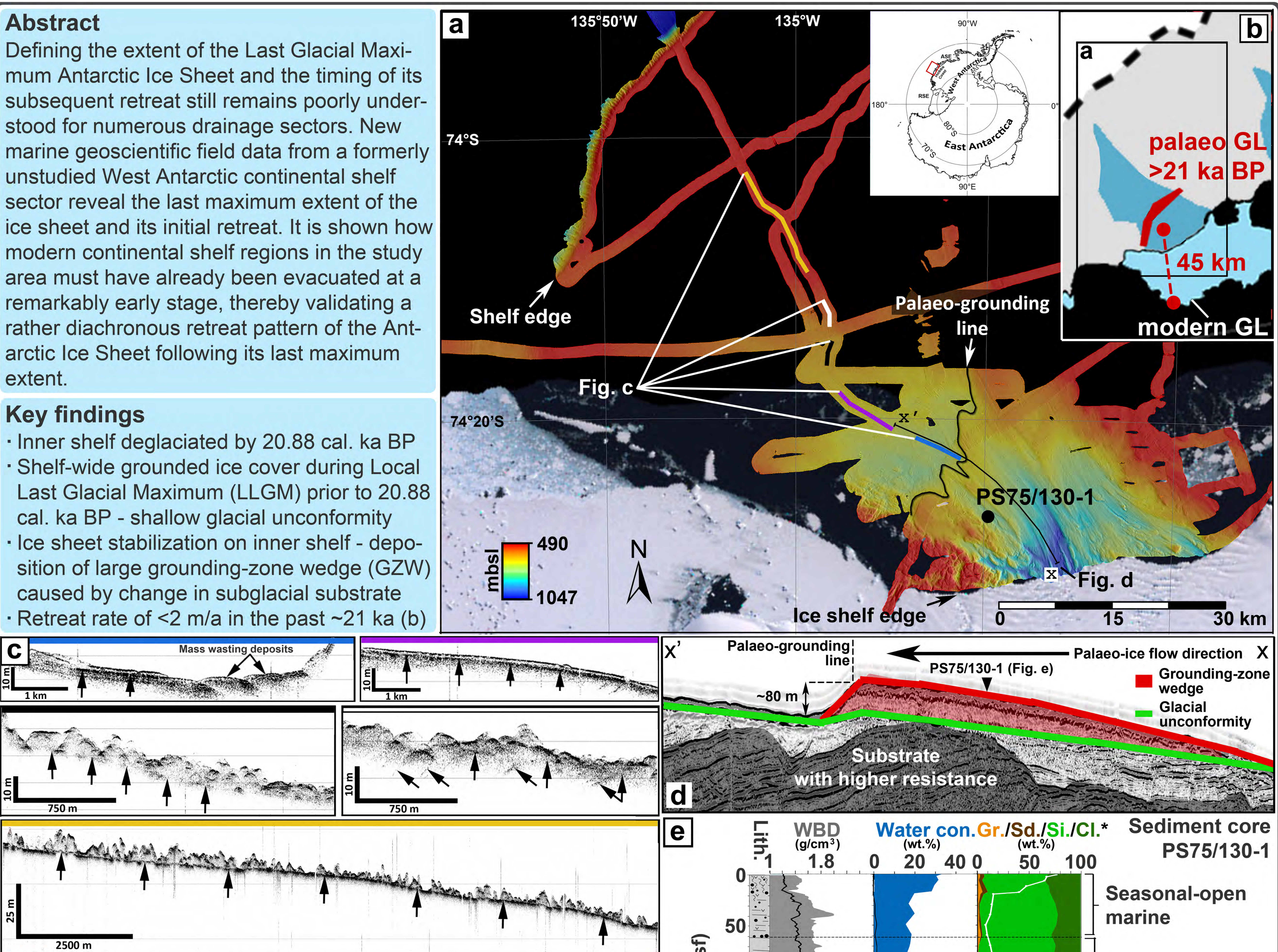

Fig. a) Glacial geomorphology

- Subglacial landforms (Mega-scale glacial lineations, drumlinoid features, and meltwater channels) only recorded on inner shelf (a) -Mid- and outer shelf largely featureless (outer shelf iceberg-scoured)

Figs. c, d) Sub-seafloor architecture - Large ( $\sim 80 \mathrm{~m}$ high) GZW covering inner shelf - Distinct glacial unconformity beneath GZW (d) - Continuation of unconformity to outer shelf (c) Fig. e) Sedimentology - Succession from sub-sea ice/sub-ice shelf deto seas.-open marine deposits (e.g. strongly scarcely laminated / scarcely $\mathbf{s t r o n g l y ~ b i o t . ~ ( e ) ~}$ \section{f}
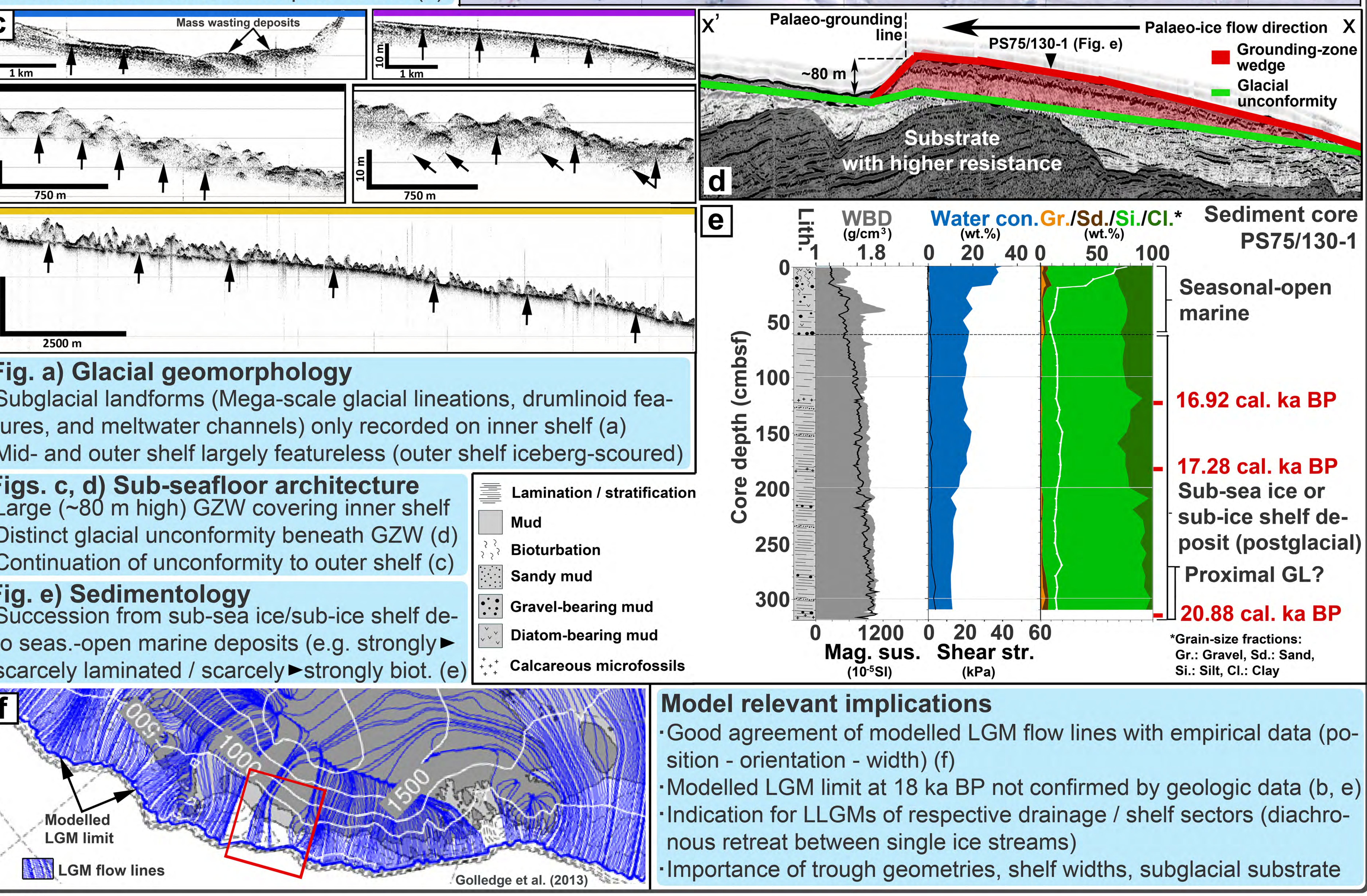

Model relevant implications

Good agreement of modelled LGM flow lines with empirical data (position - orientation - width) $(f)$

- Modelled LGM limit at 18 ka BP not confirmed by geologic data (b, e) - Indication for LLGMs of respective drainage / shelf sectors (diachronous retreat between single ice streams)

- Importance of trough geometries, shelf widths, subglacial substrate 